\title{
Neuroendocrine Tumor Arising From Meckel's Diverticulum Unexpectedly Diagnosed After Diverticulectomy and in Which Multiple Lymph Node Metastases Were Found After Reoperation: A Case Report
}

\author{
Ken Sugezawa, Hiroaki Saito, Yusuke Kono, Yuki Murakami, Yuji Shishido, Hirohiko Kuroda, Tomoyuki \\ Matsunaga, Yoji Fukumoto, Tomohiro Osaki and Yoshiyuki Fujiwara
}

Division of Surgical Oncology, Department of Surgery, School of Medicine, Tottori University Faculty of Medicine, Yonago 683-8504, Japan

\begin{abstract}
A 47-year-old man with progressive anemia possibly due to digestive tract bleeding was referred to our hospital. Contrast-enhanced computed tomography of the abdomen showed a 2-cm tumor lesion arising near the small intestine. Enteroscopy revealed a 3-cm submucosal tumor at the ileum. A gastrointestinal stromal tumor of the small intestine was suspected, and the patient underwent surgery. During the operation, a diverticulum approximately $60 \mathrm{~cm}$ orad to the terminal ileum and a tumor at the top of the diverticulum were observed. Considering the location, Meckel's diverticulum was suspected. No lymphadenopathy was present in the mesentery. Laparoscopy-assisted resection of the diverticulum without lymph node dissection was performed. The histological diagnosis of the tumor was a well-differentiated neuroendocrine tumor. Given the possibility of lymph node metastasis, we performed a second operation to remove the small intestine and lymph nodes. Histologically, 7 of the 18 dissected lymph nodes were metastatic. The postoperative course was uneventful, and the patient survived without tumor recurrence for another 2 years 8 months. Neuroendocrine tumors of Meckel's diverticulum are aggressive. Therefore, small intestinal resection along with lymph node dissection might be necessary as part of the surgical strategy.
\end{abstract}

Key words lymph node metastasis; Meckel's diverticulum; neuroendocrine tumor

Meckel's diverticulum is a congenital small intestinal diverticulum and remnant of the fetal yolk tract. ${ }^{1}$ It is

Corresponding author: Hiroaki Saito, $\mathrm{MD}, \mathrm{PhD}$

sai10@med.tottori-u.ac.jp

Received 2017 September 19

Accepted 2017 November 6

Abbreviations: CT, computed tomography; EOB-MRI, ethoxybenzyl diethylenetriamine pentaacetic acid-enhanced magnetic resonance imaging; MRI, magnetic resonance imaging; NET, neuroendocrine tumor found in up to $2 \%$ of the general population. ${ }^{2,3}$ Most Meckel's diverticula are asymptomatic, with an estimated lifetime complication rate of approximately $4 \%$. $^{4}$ Gastrointestinal bleeding is the most common clinical symptom and is generally related to an ulcer caused by secretions from the gastric mucosa. ${ }^{5}$ The incidence of tumors associated with Meckel's diverticulum is extremely low, ranging from $0.5 \%$ to $3.2 \% .^{6}$ Thirunavukarasu et al. ${ }^{7}$ found carcinoid tumors to be the most common malignancy arising from Meckel's diverticulum (76.5\% of cases), followed by adenocarcinoma (11.4\%), gastrointestinal stromal tumor/leiomyosarcoma and sarcoma (10.8\%), and lymphoma (1.3\%). These findings indicate that Meckel's diverticulum is an important location of primary neuroendocrine tumors (NETs). A consensus regarding treatment of NETs of Meckel's diverticulum is lacking because of the small number of patients with such tumors. Recent reports indicate that NETs of Meckel's diverticulum tend to be aggressive. ${ }^{8,9}$ We recently identified an NET arising from Meckel's diverticulum and herein report this case to demonstrate an appropriate treatment strategy.

\section{PATIENT REPORT}

A 47-year-old man was brought unconscious to the emergency department of another hospital. The patient had been well until approximately 2 months before this visit, when occasional diarrhea developed. He drank alcohol occasionally and did not smoke. He had no history of traumatic events and no relevant family history. Computed tomography (CT) and magnetic resonance imaging (MRI) of his head revealed no abnormal findings associated with unconsciousness. Five days later, he revisited the outpatient clinic because of tarry stool that had started after his first hospital visit. He was admitted to the hospital because of anemia as shown by a hemoglobin level of $7.6 \mathrm{~g} / \mathrm{dL}$ ( $10.1 \mathrm{~g} / \mathrm{dL}$ at his first visit). Contrast-enhanced CT of his abdomen showed a $2-\mathrm{cm}$ tumor lesion possibly arising from the small intestine (Fig. 1). He was referred to our hospital for further evaluation of the tumor. 


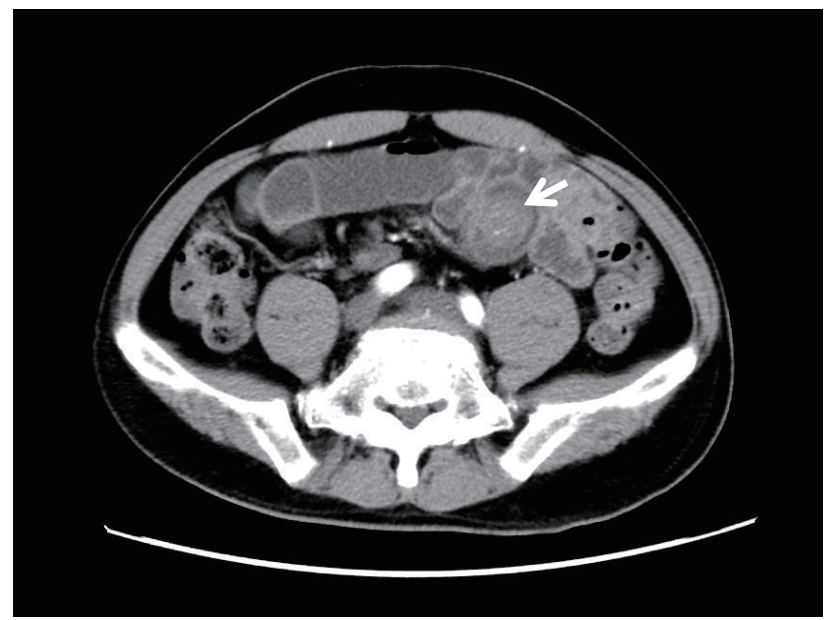

Fig. 1. Computed tomography image of the tumor. Contrast-enhanced abdominal computed tomography showed a 2-cm tumor (white arrow) possibly arising from the small intestine.

Enteroscopy revealed a 3-cm submucosal tumor at the ileum (Fig. 2). Preoperatively, we suspected a gastrointestinal stromal tumor of the small intestine. During surgery, a diverticulum was identified approximately 60 $\mathrm{cm}$ orad to the terminal ileum, and a tumor was located at the top of the diverticulum (Fig. 3). Considering its location, the diverticulum was thought to be a Meckel's diverticulum. No lymphadenopathy was observed in the mesentery. We performed laparoscopy-assisted resection of the diverticulum without lymph node dissection. The postoperative course was uneventful.

Histological examination revealed trabeculae of medium-sized polygonal cells with lightly eosinophilic cytoplasm (Fig. 4a and b). Immunohistochemical staining revealed that the tumor cells were positive for synaptophysin (Fig. 4c) and chromogranin A (Fig. 4d) and negative for cluster of differentiation 56. The MIB-1 index was $\leq 1 \%$. The histological diagnosis was a well-differentiated NET (NET G1).

Although positron emission tomography and gadolinium ethoxybenzyl diethylenetriamine pentaacetic acid-enhanced MRI (EOB-MRI) revealed no obvious metastasis, we performed small intestinal resection and lymph node dissection because of the possibility of lymph node metastasis. Surgical findings revealed two vessels near Meckel's diverticulum. These two vessels were ligated at the root to remove the lymph node. In addition, approximately $75 \mathrm{~cm}$ of the small intestine and mesentery was resected (Fig. 5). Eighteen lymph nodes were dissected, and histological examination showed that 7 were metastatic. The postoperative course was uneventful. The patient survived without recurrence for 2 years 8 months after the operation.

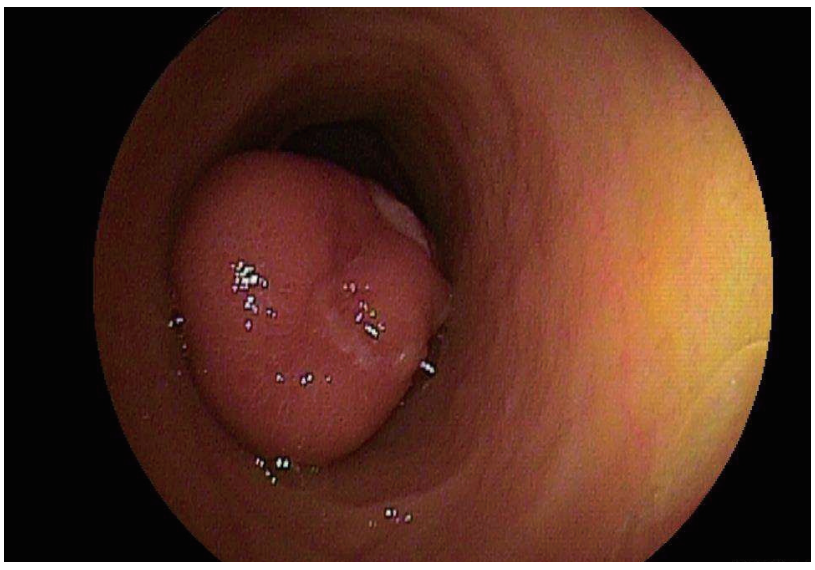

Fig. 2. Endoscopic image of the tumor. Enteroscopy revealed a 3-cm submucosal tumor at the ileum.

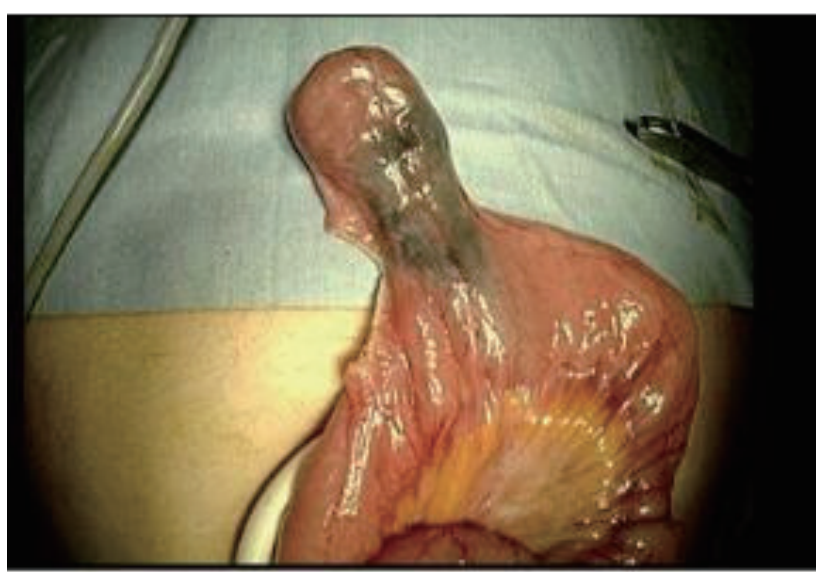

Fig. 3. Operative findings at the initial operation. Surgical findings showed a diverticulum approximately $60 \mathrm{~cm}$ orad to the terminal ileum and a tumor at the top of the diverticulum.

\section{DISCUSSION}

The incidence of NETs of Meckel's diverticulum is $<1 \%$ of all digestive NETs. However, about $10 \%$ of resected Meckel's diverticulum in adult patients may contain an NET. Therefore, the incidence of NETs is significant in patients with Meckel's diverticulum. ${ }^{8}$

Several literature reports have described NETs in a Meckel's diverticulum discovered incidentally during abdominal exploration for another indication or during autopsy. ${ }^{10-13}$ A literature review by Nies et al. ${ }^{5}$ showed that $64 \%$ of patients with NETs in a Meckel's diverticulum were asymptomatic at the time of diagnosis, and $26 \%$ of the tumors were found incidentally at autopsy. The remaining patients presented with symptoms including abdominal pain, nausea, vomiting, and diarrhea. ${ }^{5,14}$ The incidence of carcinoid syndrome in patients with ileal NETs is approximately $20 \%$ to $30 \%$, which is similar to that reported in patients with tumors arising 
(a)

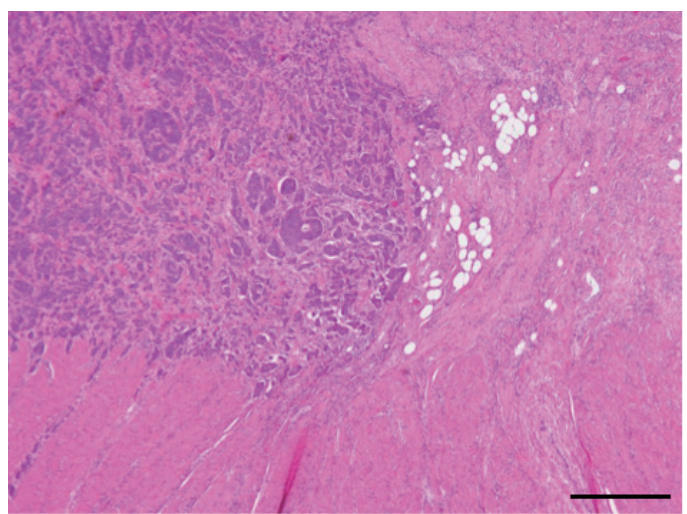

(c)

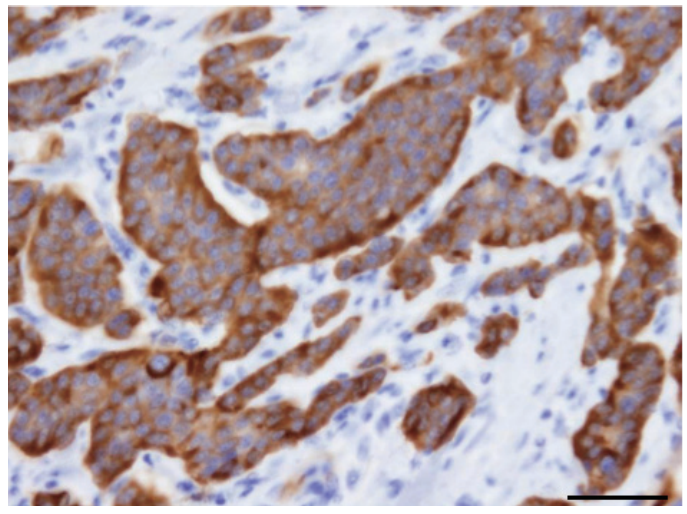

(b)

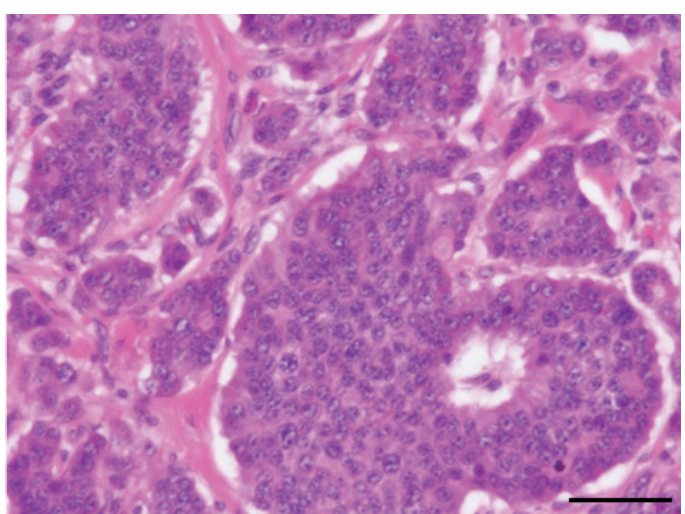

(d)

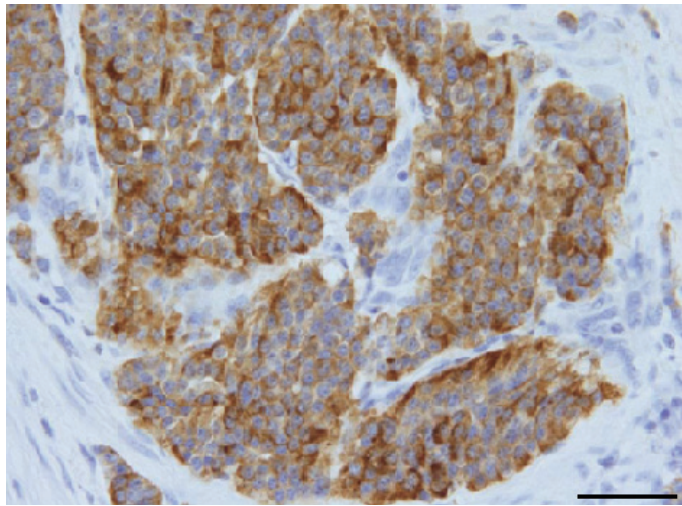

Fig. 4. Histological findings. (a, b) Tumor located at the top of the diverticulum. Nests (trabeculae) of medium-sized polygonal cells with lightly eosinophilic cytoplasm were present (hematoxylin and eosin stain). a: Bar $=500 \mu \mathrm{m}$. b: Bar $=50 \mu \mathrm{m}$. Immunohistochemical staining revealed that the tumor cells were positive for (c) chromogranin A and (d) synaptophysin. Bar $=50$ $\mu \mathrm{m}$.

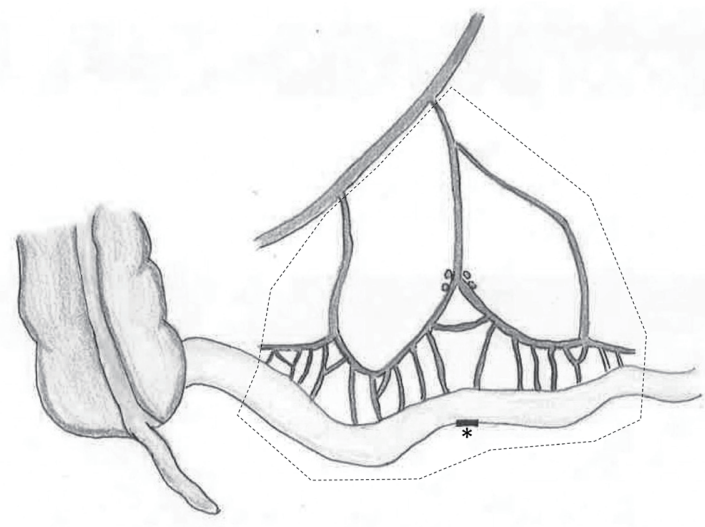

Fig. 5. Operative schema of the reoperation. Surgical findings revealed two vessels near the previous site of Meckel's diverticulum. These two vessels were ligated at the root to remove the lymph nodes located along those two vessels. In addition, approximately $75 \mathrm{~cm}$ of the small intestine and mesentery was resected. The asterisk indicates the place where Meckel's diverticulum used to be. The dotted line indicates the resection range. from a Meckel's diverticulum (12\%-25\%).8, 15, 16 Our patient exhibited progression of anemia, possibly due to bleeding from the tumor. However, because Meckel's diverticulum itself also induces bleeding, it is also possible that the anemia progressed due to bleeding from the Meckel's diverticulum. This bleeding is likely to have caused the unconsciousness with which the patient initially presented. Another possible explanation of his unconsciousness is that the NET was functional (NETs sometimes secrete insulin, which induces unconsciousness due to hypoglycemia). In this regard, although we did not perform detailed examinations because an NET was not included as a differential diagnosis before the operation, a functional NET was not likely because the patient's blood sugar level was within the normal range at his first visit to the other hospital.

Because NETs of Meckel's diverticulum are rare, their clinical and pathological characteristics are not well known. It is therefore difficult to assess the best strategy for therapeutic management. Lorenzen et al. ${ }^{9}$ 
recently described seven patients with NETs involving Meckel's diverticulum. All patients had involvement of regional nodes, including three patients with tumors of $<2 \mathrm{~cm}$ in size and four patients with liver metastases at presentation. The authors concluded that NETs arising from Meckel's diverticulum are often associated with nodal metastases and liver metastases, even when the tumors are small. ${ }^{9}$ Therefore, the optimal management of these NETs is small bowel resection with regional lymphadenectomy,and debulking of liver metastases where feasible. Poncet et al. ${ }^{8}$ also reported eight cases of NETs arising from Meckel's diverticulum. In their series, five cases were associated with mesenteric lymph node metastases and three presented with liver metastases. The authors concluded that all tumors measuring $>1 \mathrm{~cm}$ in diameter must be resected according to oncological principles. These findings emphasize the aggressive nature of NETs arising from Meckel's diverticulum.

The number of laparoscopic surgeries has been increasing worldwide, and many tumors are found incidentally after laparoscopic removal of Meckel's diverticulum. Because small bowel NETs are often multicentric, Lorenzen et al. emphasized the importance of palpating the small bowel to look for multicentric tumors. ${ }^{9} \mathrm{Be}$ cause of the aggressive nature of NETs arising from Meckel's diverticulum and the high multicentricity rate for small bowel NETs, it is inadequate to perform only diverticulectomy and laparoscopic exploration without further palpation of the bowel and mesentery. In our case, after the initial diverticulectomy, we performed a second operation involving small bowel resection with regional lymphadenectomy. During this second operation, we found metastatic lymph nodes despite the fact that no obvious metastases were revealed on positron emission tomography or EOB-MRI. Considering that the most common malignancy arising from a Meckel's diverticulum is NET, intraoperative rapid pathologic diagnosis and small bowel resection with regional lymphadenectomy should be considered at the initial operation.

In conclusion, although a standard therapeutic strategy for asymptomatic Meckel's diverticulum has not yet been established, surgeons should be aware of the risk of NETs. Considering the aggressive nature of NETs arising from Meckel's diverticulum, diverticulectomy alone might be inadequate. Small bowel resection with regional lymphadenectomy should be considered for NETs arising from Meckel's diverticulum.

Acknowledgments: We thank Angela Morben, DVM, ELS, from Edanz Group (www.edanzediting.com/ac) for editing a draft of this manuscript.

The authors declare no conflict of interest.

\section{REFERENCES}

1 Kado K. Case of ileus due to inverted Meckel's diverticulum. Yonago Acta Med. 1966;10:207-10. PMID: 5984710.

2 Yahchouchy EK, Marano AF, Etienne JC, Fingerhut AL. Meckel's diverticulum. Journal of the American College of Surgeons. 2001;192:658-62. PMID: 11333103.

3 Levy AD, Hobbs CM. From the archives of the AFIP. Meckel diverticulum: radiologic features with pathologic Correlation. Radiographics : a review publication of the Radiological Society of North America, Inc. 2004;24:565-87. PMID: 15026601.

4 Soltero MJ, Bill AH. The natural history of Meckel's Diverticulum and its relation to incidental removal. A study of 202 cases of diseased Meckel's Diverticulum found in King County, Washington, over a fifteen year period. American journal of surgery. 1976;132:168-73. PMID: 952346.

5 Nies C, Zielke A, Hasse C, Ruschoff J, Rothmund M. Carcinoid tumors of Meckel's diverticula. Report of two cases and review of the literature. Diseases of the colon and rectum. 1992;35:589-96. PMID: 1587179.

6 Ymaguchi M, Takeuchi S, Awazu S. Meckel's diverticulum. Investigation of 600 patients in Japanese literature. American journal of surgery. 1978;136:247-9. PMID: 308325.

7 Thirunavukarasu P, Sathaiah M, Sukumar S, Bartels CJ, Zeh $\mathrm{H}$, 3rd, Lee KK, et al. Meckel's diverticulum--a high-risk region for malignancy in the ileum. Insights from a population-based epidemiological study and implications in surgical management. Annals of surgery. 2011;253:223-30. PMID: 21135700.

8 Poncet G, Hervieu V, Walter T, Lepinasse F, Chardon L, Pilleul F, et al. Neuroendocrine tumors of Meckel's diverticulum: lessons from a single institution study of eight cases. Journal of gastrointestinal surgery : official journal of the Society for Surgery of the Alimentary Tract. 2011;15:101-9. PMID: 20824383.

9 Lorenzen AW, O'Dorisio TM, Howe JR. Neuroendocrine tumors arising in Meckel's diverticula: frequency of advanced disease warrants aggressive management. Journal of gastrointestinal surgery : official journal of the Society for Surgery of the Alimentary Tract. 2013;17:1084-91. PMID: 23558715.

10 Anderson DJ. Carcinoid tumor in Meckel's diverticulum: laparoscopic treatment and review of the literature. The Journal of the American Osteopathic Association. 2000;100:432-4. PMID: 10943090.

11 Leinati A, Iacovoni P, Cavallero G, Dealessi M, Perrone F, Zanardi F, et al. [Carcinoid of Meckel's diverticulum]. Minerva chirurgica. 1995;50:501-4. PMID: 7478064.

12 Mudatsakis N, Paraskakis S, Lasithiotakis K, Andreadakis E, Karatsis P. Acute appendicitis and carcinoid tumor in Meckel's diverticulum. Three pathologies in one: a case report. Techniques in coloproctology. 2011;15 Suppl 1:S83-5. PMID: 21887558.

13 Weitzner S. Carcinoid of Meckel's diverticulum. Report of a case and review of the literature. Cancer. 1969;23:1436-40. PMID: 5771072.

14 Modlin IM, Shapiro MD, Kidd M. An analysis of rare carcinoid tumors: clarifying these clinical conundrums. World journal of surgery. 2005;29:92-101. PMID: 15599742.

15 Silk YN, Douglass HO, Jr., Penetrante R. Carcinoid tumor in Meckel's diverticulum. The American surgeon. 1988;54:6647. PMID: 3056140.

16 Weber JD, McFadden DW. Carcinoid tumors in Meckel's diverticula. Journal of clinical gastroenterology. 1989;11:682-6. PMID: 2685098. 